- peer-reviewed papers face myriad hurdles, from publisher copyrights to disparate websites that make bulk-downloading difficult. "We're trying to think of preprints as data," says Vale. It would be both technically and legally straightforward for computers to crawl through the collection of preprints on the central site, where they would appear under an open-access licence.

Polka would not say how much ASAPbio expects the site to cost, but arXiv funding totals about US $\$ 925,000$ a year, paid for by a global collective of more than 200 research institutions and funders. Ginsparg says expenses for the life-sciences site should be around $\$ 5$ a manuscript, once it is publishing tens of thousands of manuscripts each year. Funders who support the site have not yet committed to paying for it, but Kiley expects that they will do so once details have been hammered out.
Other funders that have come out in support of the central service include the UK Medical Research Council, the Howard Hughes Medical Institute (HHMI), the Canadian Institutes of Health Research and the European Research Council. "That's going to send a strong message to the science community that this kind of communication is encouraged," says Vale.

\section{CULTURAL CHALLENGE}

Jason Hoyt, chief executive of the journal PeerJ (which also operates a preprint service), says he supports a central preprint site and that his company might bid to help create it. But such a site will succeed only if it can induce a large proportion of life scientists to view preprints as the dominant currency for career progression, he says. "The challenge is to overturn the thinking in biology."

ASAPbio and the funders supporting a central preprint service emphasize that it's no replacement for peer-reviewed journals. They note that the vast majority (more than $80 \%$ in some fields) of arXiv posts wind up in journals. "We really see this as a complement to the journal system, rather than anything that could be threatening," says Polka, who adds that a central service will not attempt to organize peer review.

That would be a missed opportunity, says Rebecca Lawrence, managing director of London-based F1000Research, which posts papers before they are peer reviewed (but does not consider these as preprints). She would like to see peer review occur through a central preprint service, thereby reducing the influence that traditional journals have on scientists' careers.

"It's a great shift in the right direction," she says, "but I think we need to go a lot further." -

\title{
Elusive triangulene created by moving atoms one at a time
}

Researchers used microscope tip to make unstable hydrocarbon with 'molecular surgery'.

\section{BY PHILIP BALL}

$\mathrm{R}$ esearchers at IBM have created an elusive molecule by knocking around atoms using a needle-like microscope tip. The flat, triangular fragment of a mesh of carbon atoms, called triangulene $e^{1}$, is too unstable to be made by conventional chemical synthesis, and could find use in electronics.

This isn't the first time that atomic manipulation has been used to create unstable molecules that couldn't be made conventionally — but this one is especially desirable. "Triangulene is the first molecule that we've made that chemists have tried hard, and failed, to make already," says Leo Gross, who led the IBM team at the firm's laboratories in Zurich, Switzerland.

The creation of triangulene demonstrates a new type of chemical synthesis, says Philip Moriarty, a nanoscientist who specializes in molecular manipulation at the University of Nottingham, UK. In conventional synthesis, chemists react molecules together to build up larger structures. Here, by contrast, atoms on individual molecules were physically manipulated using a microscope.

But making molecules one at a time will be useful only in particular situations. And

\section{RADICAL TRIANGLE}

Triangulene is a flat molecule made up of a hexagonal mesh of carbon and hydrogen atoms (left). IBM researchers made the molecule by manipulating atoms with a scanning probe microscope, and then imaged it (right).

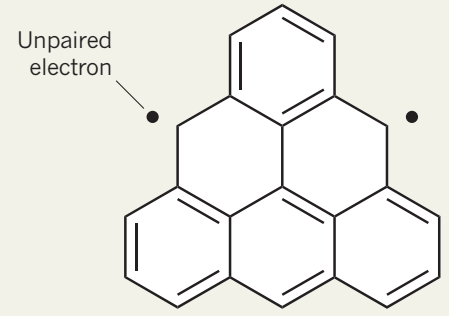

the method is unlikely to work for those with complicated shapes or structures.

Triangulene is similar to a fragment of graphene, the atom-thick material in which carbon atoms are joined in a hexagonal mesh. The new molecule is made up of six hexagons of carbon joined along their edges to form a triangle, with hydrogen atoms around the sides (see 'Radical triangle'). Two of the outer carbon atoms contain unpaired electrons that can't pair up to make a stable bond.

Such a molecule is highly unstable because the unpaired electrons tend to react with anything around them. "As soon as you synthesize it, it will oxidize," says Niko Pavliček, a member of the IBM team. So far, the closest conventional synthesis has come to making molecules of this sort involves buffering the reactive edges with bulky hydrocarbon appendages ${ }^{2}$.

The IBM team turned to a scanning probe microscope, which has a needle-sharp tip that 'feels' a material's shape. The technique is usually used to image molecules, by measuring attractive forces between the tip and sample, or the electric currents that pass between them. The IBM team has shown ${ }^{3}$ that, if the tip has a small molecule such as carbon monoxide attached to it, force microscopy can provide images of such high resolution that they resemble the ball-andstick diagrams of chemistry textbooks.

Gross's team has already demonstrated ${ }^{4}$ 
how the microscope can be used to direct the course of chemical reactions and make unstable 'intermediate' molecules. To produce triangulene, the team began with a precursor molecule called dihydrotriangulene, which lacks the reactive unpaired electrons.

The researchers deposited these molecules on a surface - salt, solid xenon and copper are all suitable - and inspected them under the microscope. They then used two successive voltage pulses from the tip, carefully positioned above the molecules, to blast off two hydrogen atoms and create the unpaired electrons. The work is published in Nature Nanotechnology ${ }^{1}$.

The team then imaged the products with the microscope, first picking up a carbon monoxide molecule to acquire the high resolution. The images had the shape and symmetry predicted for triangulene. Under the highvacuum, low-temperature conditions of the experiments, the molecules remained stable for as long as the researchers looked.

"To my knowledge, this is the first synthesis of unsubstituted triangulene," says chemist Takeji Takui of Osaka City University in Japan, who has previously synthesized triangulenetype molecules ${ }^{2}$.

Moriarty calls the work elegant, but is surprised that triangulene remained stable on a copper surface, where he might have expected it to react with the metal. In one set of experiments, says Pavliček, the molecule was still sitting on the copper four days after the team made it.

The researchers also probed triangulene's magnetic properties. They found that, as they had expected, the two unpaired electrons have aligned spins - the quantum-mechanical property that gives electrons a magnetic orientation.

This could make triangulene useful in electronics, they say. Takui agrees, and foresees applications in quantum computing, quantum information processing and a field known as spintronics, in which devices manipulate electron spins to encode and process information.

Making molecules one at a time might not seem very promising, but Gross points out that current quantum computers use only a handful of quantum bits, or qubits, each of which could correspond to a single molecule. Even if you

\section{CORRECTION}

The Editorial 'Keep science on track' (Nature

542, 137; 2017) wrongly attributed to Till Sawala and then to Nadine El-Enany the opinion that the proposed boycott of US-based conferences is intended to demonstrate against a ban that hurts everyone. The article also misquoted Nadine El-Enany (originally incorrectly attributed to Sawala) as saying a need to make 100 such molecules "by hand", he says, "it would be worth going through that manual labour".

And although it's not clear how easily the approach could be applied to molecules that aren't flat, Gross says that such atom manipulation can be performed for 3D molecules to some extent.

Even with triangulene and related graphenelike fragments, "there's a lot of exciting science still to be done", says Moriarty. The IBM team "continues to set a high bar for the rest of us", he adds.

1. Pavliček, N. et al. Nature Nanotechnol. http://dx.doi. org/10.1038/nnano.2016.305 (2017).

2. Morita, Y., Suzuki, S., Sato, K. \& Takui, T. Nature Chem. 3, 197-204 (2011).

3. Gross, L., Mohn, F., Moll, N., Liljeroth, P. \& Meyer, G. Science 325, 1110-1114 (2009).

4. Pavliček, N. et al. Nature Chem. 7, 623-628 (2015).

petition she co-organized aimed to stop more countries being added to the blacklist. In fact, El-Enany had referred to concerns about people who are not from the seven Muslimmajority countries stipulated in the travel ban, but who are nevertheless facing detention and harassment at the border. Sawala had referred to the unsustainable cost of the ban on science and on the life of scientists. 